See discussions, stats, and author profiles for this publication at: https://www.researchgate.net/publication/318737993

\title{
Fractional order admittance control for physical human-robot interaction
}

Conference Paper · June 2017

DOI: 10.1109/WHC.2017.7989911

CITATIONS

2

4 authors:

(2) Yusuf Aydin

1. Koc University

3 PUBlications 3 CitATIONS

SEE PROFILE

Volkan Patoglu

4. Sabanci University

140 PUBLICATIONS 1,032 CITATIONS

SEE PROFILE
READS

32

Ozan Tokatli

University of Reading

13 PUBliCATIONS 13 CITATIONS

SEE PROFILE

Cagatay Basdogan

Koc University

123 PUBLICATIONS 3,668 CITATIONS

SEE PROFILE

Some of the authors of this publication are also working on these related projects:

Project ASSISTON-GAIT: An Overground Gait Trainer with an Active Pelvis-Hip Exoskeleton View project

haptics View project 


\title{
Fractional Order Admittance Control for Physical Human-Robot Interaction
}

\author{
Yusuf Aydin ${ }^{1}$, Ozan Tokatli ${ }^{2}$, Volkan Patoglu ${ }^{3}$ and Cagatay Basdogan ${ }^{1}$
}

\begin{abstract}
In physical human-robot interaction (pHRI), the cognitive skill of a human is combined with the accuracy, repeatability and strength of a robot. While the promises and potential outcomes of pHRI are glamorous, the control of such coupled systems is challenging in many aspects. In this paper, we propose a new controller, fractional order admittance controller, for pHRI systems. The stability analysis of the new control system with human in-the-loop is performed and the interaction performance is investigated experimentally with 10 subjects during a task imitating a contact with a stiff environment. The results show that the fractional order controller is more robust than the standard admittance controller and helps to reduce the human effort in task execution.
\end{abstract}

\section{INTRODUCTION}

Repeatability and accuracy of robots are, typically, superior to humans; however, the problem solving skills of humans are unmatched. Integrating the benefits of human and robot into tasks involving pHRI may result in a significant increase in the overall performance. Such collaborative tasks require human and robot to be in the same environment and interact with each other throughout the operation. For instance, human driving a robot to position an object, while robot bares its weight, is an example for such a collaborative task. In such a scenario, the robot has to guarantee the safety of the human under changes in both environmental conditions and/or human dynamical behavior, while helping human to achieve a high task performance. Seeming trivial, the contradicting nature of safety and performance creates a challenge for researchers.

In this paper, we propose a fractional order admittance controller (FOAC), for pHRI. This control scheme utilizes the fractional order calculus, which allows the use of integrators/differentiators of arbitrary orders. Hence, apart from adjusting the usual controller parameters, by changing the order of integration, one can change the output response characteristics of the closed-loop system. In this paper, the stability of such a controller and its effect on human task performance are investigated for a pHRI task.

\section{A. Physical Human Robot Interaction}

In the literature, a special emphasis is given to the stability of pHRI systems. The presence of human in the control

\footnotetext{
${ }^{1}$ Yusuf Aydin and Cagatay Basdogan are with College of Engineering, Koc University, Sariyer Istanbul, 34450, Turkey \{yaydin, cbasdogan\}eku.edu.tr

${ }^{2}$ Ozan Tokatli is with Biomedical Engineering, University of Reading, Whiteknights RG6 6AX, UK o.tokatlidreading.ac.uk

${ }^{3}$ Volkan Patoglu is with Mechatronics Engineering, Sabanci University, Orhanli Tuzla Istanbul 34956, Turkey vpatogluesabanciuniv.edu
}

loop creates an additional difficulty in stability analysis. This stability analysis, named coupled stability since the human operator is coupled with the rest of the closed-loop system, is challenging due to the lack of information on modeling human dynamical behavior which is time and configurationdependent, and also nonlinear. On the other hand, as it has been observed in the literature, the human model, specifically the stiffness component, has a direct effect on the coupled stability. Tsumugiwa et al. [1] use the root-locus technique to determine the stability of a closed-loop control system designed for a pHRI task. It has been observed that the changes in the environment stiffness significantly contribute to the instability of the system. Duchaine et al. [2] estimate human arm stiffness experimentally and then use this information in Lyapunov analysis for determining the critical damping of the closed-loop system. Besides being off-line, this approach neglects the time-varying changes in human force response and focuses on the maximum stiffness only. In [3], human arm model is estimated on-line using EMG sensors. Depending on the arm stiffness, authors adjust the controller gains on-the-fly to ensure stability. However, this method requires attaching sensors on human operator, which is not practical.

The coupled stability analysis of the pHRI system in the absence of human model can be conducted using the passivity framework [4]. This approach assumes that human operator is a passive network element in the closed-loop system and does not generate energy. Therefore, if the rest of the control system can be guaranteed to be passive, one can argue that the closed-loop system is also passive, so, the coupled stability is ensured. In this approach, stability is guaranteed for large changes in the environmental impedance. However, non-passive (i.e. active) systems are not always unstable [5]. This is due to the fact that not every system is exposed to such large changes in the environment. For instance, although the dynamics of human arm changes over time, human arm impedance vary in a limited range [6], [7]. Knowing that human arm impedance is bounded, Buerger and Hogan [8] propose a complementary stability approach, and design a controller which robustly maintains stability in this bounded range. This approach relaxes the conservative constraints of the passivity approach. Similarly, Haddadi and Hashtrudi-Zaad [9] propose a method which guarantees stability in a less conservative manner when the bounds of the environmental impedance are known.

All the studies above investigate and improve the stability of pHRI systems using standard admittance/impedance controllers (i.e. integer order admittance/impedance controllers). 
In this study, we propose a fractional order admittance controller for pHRI, which is designed with the assumption that human arm dynamics can be described by a second order and bounded model. The proposed controller exhibits more robust behavior to the variations in the human arm stiffness when it is compared to an integer order admittance controller (IOAC). In addition, an analysis of the closed-loop impedance is provided for a better insight on the frequencybased characteristics of the controller. We also show through pHRI experiments that the proposed controller improves interaction performance.

\section{PRELIMINARIES}

\section{A. Fractional Order Control}

Fractional order calculus allows the use of differentiation and integration of arbitrary order. Among many application areas, fractional order calculus has been utilized in modeling of viscoelastic materials and for motion control of robotic systems. An impedance controller using fractional order calculus for haptic rendering of virtual walls can be found in [10]. In this study, the Grunwald-Letnikov formulation is used for calculation of fractional order derivative/integral (differintegral) since this formulation allows easier implementation of the differintegral in discrete time domain [11].

\section{B. Characterization of the Robot}

In our experiments, subjects interact with UR5 robot (Universal Robots Inc.) to perform a pHRI task. The dynamical model of the robot is not provided by the manufacturer. For this reason, the dynamical behavior of the robot is characterized experimentally along horizontal axis for a specific joint configuration. Thus, a transfer function model of the robot, $G(s)$, is estimated by using recorded $(V(s))$ and reference $\left(V_{\text {ref }}(s)\right)$ speeds, where $G(s)=V(s) / V_{\text {ref }}(s)$.

\section{Human Arm Impedance}

The main challenge in pHRI is the presence of human operator, who display a time-varying and nonlinear behavior which is difficult to model. On the other hand, a simple linear model is useful to investigate the dynamics of interaction under a proposed controller. In this study, we have used a linear second order impedance transfer function to model human operator.

$$
Z_{h}(s)=\frac{m_{h} s^{2}+b_{h} s+k_{h}}{s}
$$

In this equation, $m_{h}, b_{h}, k_{h}$ represent human arm mass, damping, and stiffness, respectively.

\section{Control Architecture}

Fig. 1 shows the admittance control architecture used in this study. In this architecture, human $Z_{h}(s)$ and environment $Z_{e}(s)$ are assumed to be coupled $\left(Z_{c}(s)=Z_{h}(s)+Z_{e}(s)\right)$, and the resultant interaction force $F_{I}$ is measured by the force sensor attached to the end-effector of robot. This measurement is filtered by a low pass filter $H(s)$ and sent to the admittance controller, $Y(s)$. The controller generates the

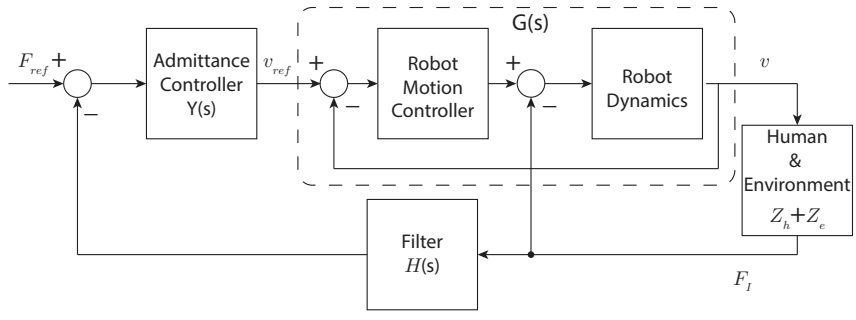

Fig. 1: Control architecture of the HRI system

corresponding reference velocity $v_{r e f}$. Then, robot's motion controller transmits sufficient torque to its joints in order to achieve the desired (reference) velocity. The robot's motion controller is robust to the environmental forces acting on its end-effector and it is assumed that the motion of the robot is not affected by these forces. The transfer function of the closed loop system is given as:

$$
T(s)=\frac{V(s)}{F_{\text {ref }}(s)}=\frac{Y(s) G(s)}{1+Y(s) G(s) Z_{c}(s) H(s)}
$$

Using the control architecture given in Fig. 1, we compare two admittance controllers in terms of stability and closed loop impedance characteristics;

1) Integer order admittance controller: IOAC has been already used in earlier pHRI studies in the following form:

$$
Y(s)=\frac{1}{m s+b}
$$

where, $m$ and $b$ are admittance mass and damping, respectively.

2) Fractional order admittance controller: FOAC utilizes fractional order integrator with order $\alpha$ in the controller as in (4). In this study, the range of the integration order is $0<\alpha \leq 1$. As the integration order is decreased gradually, the energy storage characteristics of the controller decay, and energy dissipation characteristics increase. For $\alpha=0$, the controller acts like a damper. On the other hand, when the integration order is $\alpha=1$, FOAC becomes IOAC. Therefore, the mass and damping supplied by the controller to the system change depending on the integration order.

$$
Y(s)=\frac{1}{m s^{\alpha}+b}
$$

\section{ANALysis OF THE CONTROLleR}

This section investigates the coupled stability characteristics of the proposed FOAC and compares it with IOAC. Following the stability analysis, the performances of the two systems are investigated using the effective impedance analysis [12], [13]. In this paper, we have chosen a single integration order value ( $\alpha=0.7$ ) for FOAC to investigate its effect on stability and performance. Even though having a particular integration order cannot represent all aspects of FOAC, it provides a basis to compare IOAC and FOAC in the pHRI context.

\section{A. Stability Analysis}

For a safe pHRI application, one has to guarantee the coupled stability of the closed-loop system. In this section, 


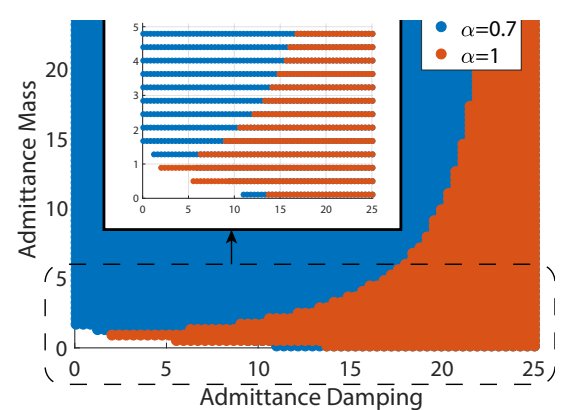

(a) Stability map of the pHRI system for integration order $\alpha \in\{0.7,1\}, m_{h}=1.5 \mathrm{~kg}, b_{h}=15$ $\mathrm{Ns} / \mathrm{m}$, and $k_{h}=610 \mathrm{~N} / \mathrm{m}$.

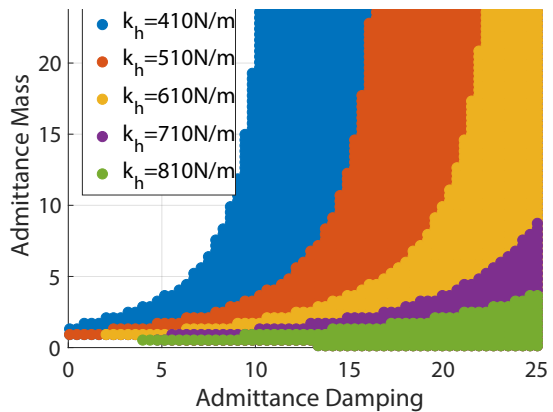

(b) Stability map of the pHRI system under IOAC for stiffness values $k_{h} \in\{410-810\} \mathrm{N} / \mathrm{m}$, and $\alpha=1$.

Fig. 2: Stability maps.

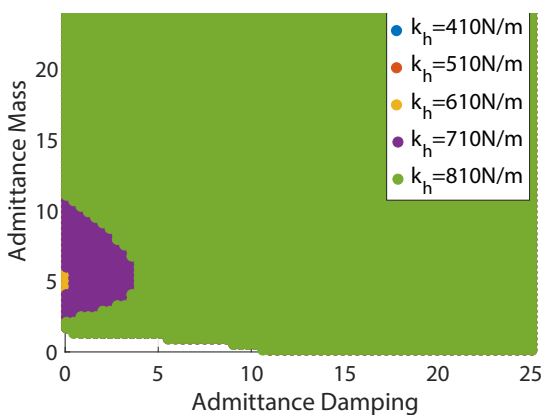

(c) Stability map of the pHRI system under FOAC for stiffness values $k_{h} \in\{410-810\} \mathrm{N} / \mathrm{m}$, and $\alpha=0.7$. the stability characteristics of the coupled system is analyzed for different controller parameters using stability maps. A stability map is a graphical representation of the parameter values where the resulting closed-loop system is stable. In this section, a comparative analysis of the stability maps for both IOAC and FOAC are given. The stability analysis of the system is performed by checking the location of the closedloop poles. Since characteristic equations of fractional order systems are no longer polynomials, we use a transformation from fractional order to integer order domain according to Matignon's theorem [11], [14]. Hence, resulting integer order system has equivalent stability characteristics as the fractional order system.

Two different analyses are performed to compare the stability characteristics of the proposed controller with its integer order counterpart. First, the stability maps for FOAC ( $\alpha=0.7)$ and IOAC $(\alpha=1)$ are constructed for the maximum value of human arm stiffness reported in [6], [7] to investigate the effect of integration order, $\alpha$. The second analysis focuses on investigating the robustness of each controller for a range of stiffness values. In these analyses, we assume that human always maintains his/her contact with the end-effector of the robot. In such a condition, mass and damping values in human arm model would not change significantly, whereas arm stiffness may change drastically due to the cocontraction of muscles. Hence, we are investigating robustness of the proposed controller to stiffness changes since it has been reported that changes in human arm stiffness significantly contributes to the instabilities observed in pHRI [1], [15]. The stability analysis is conducted for a range of human arm stiffness values, and typical values of $b_{h}=15 \mathrm{Ns} / \mathrm{m}$ and $m_{h}=1.5 \mathrm{~kg}$ are used for human arm damping and mass, respectively, as reported in [6], [7].

1) Effect of Integration Order: In this analysis, the integration order of the admittance controller is changed while keeping the other parameters, $m_{h}, b_{h}$, and $k_{h}$ of the closedloop system fixed.

It can be observed from Fig. 2a that FOAC extends the area of the stable region for the set of parameters used in human arm model. When $\alpha<1$, more damping is supplied to the system resulting in a larger region for stability.

2) Effect of Human Arm Stiffness: In our analysis, the stiffness of human arm is assumed to be coupled with that of the environment for simplification. For this reason, a larger range of values are utilized for human arm stiffness $k_{h}$ in the analysis. Fig. $2 b$ shows the stability map for IOAC for stiffness $k_{h} \in\{410-810 \mathrm{~N} / \mathrm{m}\}$. The destabilizing nature of the human arm stiffness can be observed from Fig. 2b; as the arm stiffness increases, the area for which the closedloop system is stable shrinks. Moreover, it can be seen that higher admittance damping is required to stabilize the system for higher values of arm stiffness. The same analysis is conducted for FOAC and the resulting stability map is shown in Fig. 2c. The change in the stability map of FOAC for varying human arm stiffness is minimal compared to that of IOAC.

The stability maps suggest that FOAC is more robust to the changes in human arm stiffness. Of course, our analyses are valid for a second order LTI model of human arm impedance for the given parameter values.

\section{B. Effective Impedance Analysis}

The stability analysis of the proposed FOAC shows that FOAC is more robust to changes in human arm stiffness than IOAC. This section investigates the effective closed loop impedance of our system and provides further insight on how robustness is improved under FOAC.

Impedance reflected to human can be decomposed into effective stiffness $\left(E S(\omega)=\omega \Im^{-}\{Z(j \omega)\}\right)$, mass $(E M(\omega)=$ $\left.\omega^{-1} \Im^{+}\{Z(j \omega)\}\right)$ and damping $\left(E D(\omega)=\Re^{+}\{Z(j \omega)\}\right)$, as suggested in [12], [13], where, $Z(j \omega)=F_{h}(j w) / V(j w)$ is closed loop impedance transfer function, and $F_{h}$ is the force applied by human.

In the analysis (and also for the experiments reported in Section IV), a stiffness value of $610 \mathrm{~N} / \mathrm{m}$ is used for the spring, which represents the environment. The admittance mass and damping values of the controller are set to $m=\{5,20\} \mathrm{kg}$, and $b=25 \mathrm{Ns} / \mathrm{m}$. These values are chosen from the stable region in the stability maps (Fig. 2a). The changes in effective stiffness, mass, and damping are shown in Fig. 3a-c. The effective mass is zero up to the natural frequency $\left(w_{n}\right)$ since stiffness dominates the dynamical behavior in that range. When $w>w_{n}$, the effective mass for IOAC is higher than that of FOAC. The effective stiffness is higher under FOAC than that of IOAC for $w<w_{n}$. Afterwards, it becomes zero since mass dominates the behavior of the system for $w>w_{n}$. Natural frequency of the system under FOAC is higher than that of IOAC since higher effective stiffness and lower effective mass are rendered under FOAC. As expected, the magnitude 


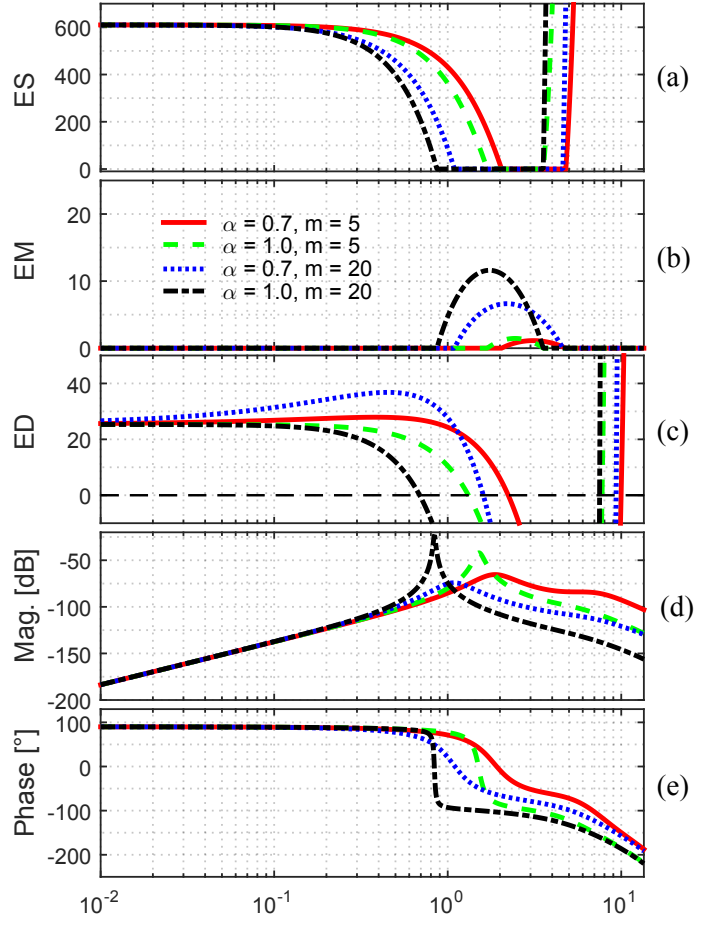

Fig. 3: Effective stiffness (a), mass (b) and damping (c) of the impedance transfer function, magnitude (d) and phase (e) plot of the closed loop transfer function.

and phase plots (Fig. 3d-e) of the closed loop system also show that natural frequency is higher under FOAC.

Effective damping analysis reveals an interesting result: damping supplied by the FOAC remains higher for a larger range of frequencies, which is beneficial from the robustness point of view (Fig. 3c). Furthermore, low (high) damping is rendered for lower (higher) operating frequencies up to a certain frequency under FOAC, whereas effective damping decays with the frequency under IOAC. This frequencydependent damping behavior of FOAC can be useful for reducing vibrations in pHRI tasks involving contact interactions with environment.

\section{EXPERIMENT}

In pHRI, maximizing task performance while maintaining stability is desired. The effective impedance analysis shows that damping under FOAC remains effective for a larger range of frequencies, which is desirable for stable interactions. This feature of FOAC may improve human performance in pHRI tasks involving especially contact interactions with environment. For instance during a needle insertion task, a needle connected to the end-effector of a robot penetrates into soft tissue under the guidance of human operator while the robot keeps the needle on a trajectory. In this scenario, high damping for higher frequencies reduces vibrations during the initial penetration or the later stages of guiding the needle through different tissue layers while low damping for lower frequencies may allow human to position the robot and bring it to the target more easily with less effort. An experiment is designed to further investigate this frequency-dependent damping feature of FOAC, and effect of admittance controller (i.e. FOAC vs IOAC) and mass on human performance in a pHRI task.

\section{A. Experimental Procedure}

In our experimental setup, a spring $(k=610 \mathrm{~N} / \mathrm{m})$ is connected between a fixed support and the end-effector of UR5 robot (Fig. 4a). The subject grasps the handle between spring and the robot to guide the end-effector and compress the spring. The spring simply represents the environment in which the robot guided by a human operator is in continuous contact as in drilling a wall or inserting a needle to soft tissue. In our experiments, the subjects are asked to compress the spring up to a certain position (ramp phase, Fig. 4b), and then, hold it at that position for 10 seconds (hold phase, Fig. 4c). For visual feedback to the subjects, a virtual object (black rectangle, labeled as "O" in Fig. 4b) having a finite size is displayed on the computer screen and its movement is virtually linked to that of the end-effector guided by the subject. The motion of the end-effector is constrained to stay on a straight path, and the admittance controller scheme described in Section II-D is utilized. In order to make the compression rate of the spring equal for each subject, a visual cursor (i.e. blue rectangle, labeled as "C") moving with a constant speed $\left(\mathrm{v}_{c}=0.5 \mathrm{~cm} / \mathrm{s}\right)$ is also displayed on the computer screen. The cursor stops when it reaches the end of the ramp phase, labeled as "hold position". Following, the subject is required to hold the spring at this position for 10 seconds (hold phase) to complete the task (Fig. 4c).

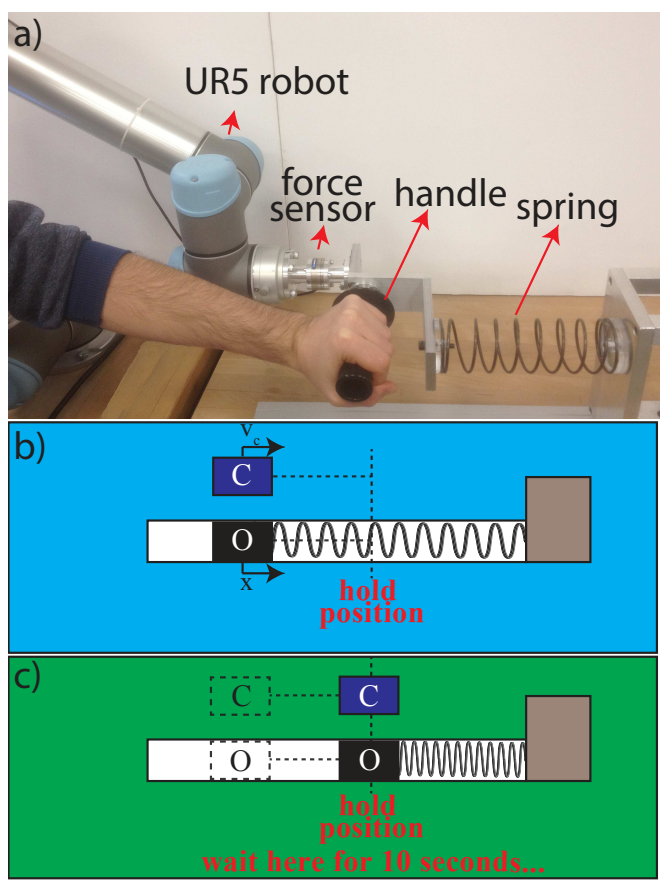

Fig. 4: The experimental setup and visual feedback provided to the subjects during the experiments.

We conduct an experiment to compare subjects' performance under IOAC $(\alpha=1)$ and FOAC $(\alpha=0.7)$. Since admittance mass is the coefficient in front of the integrator in admittance controller, we also investigate its effect on 
these controllers for low (i.e. LAM, $m=5 \mathrm{~kg}$ ), and high (i.e. HAM, $m=20 \mathrm{~kg}$ ) admittance mass. The admittance damping $(b=25 \mathrm{Ns} / \mathrm{m})$ is kept constant throughout the experiment. These values are chosen by considering the stable regions of the stability maps constructed for $k=610$ $\mathrm{N} / \mathrm{m}$ (Fig. 2a). Hence, four different conditions (FOACLAM, IOAC-LAM, FOAC-HAM, IOAC-HAM) are tested in this experiment. Each condition is repeated 9 times. Thus, there are $36(4 \times 9)$ trials in the experiment, which are displayed to the subjects in random order. Prior to the actual experiment, each subject is given a training session to get him/her familiar with the setup. 10 subjects (5 males and 5 females, average age $28 \pm 5$ ) have participated in this experiment. The subjects gave informed consent about their participation in the experiment.

\section{B. Data Collection and Metrics}

In our experiment, robot constrains the motion of subjects along a horizontal line while they compress a spring. For simplicity, we assume that the robot perfectly follows the desired trajectory generated by the admittance controller, and the force applied by the subject is the sum of the forces for compressing the spring $\left(F_{S}\right)$ and generating the motion trajectory of the robot (i.e. interaction force, $F_{I}$ ). The task requirements (i.e. compressing the spring) are not changing during the experiment, so, it can be assumed that $F_{S}=k \delta x$ ( $\delta x$ is the amount of compression) is not influenced by the controller or admittance mass. On the other hand, $F_{I}$ is the interaction force that is measured by a force sensor (Mini40, ATI Inc.) and fed back to the admittance controller. Hence, by inspecting this force, we can deduce how force applied by human is influenced by the changes in admittance controller and admittance mass. All the metrics evaluated in this section are based on this interaction force unless otherwise is specified. We use average interaction force $\left(F_{\text {ave }}=1 /\left(t_{f}-t_{i}\right) \int_{t_{i}}^{t_{f}}\left|F_{I}(t)\right| d t\right)$, and average power $\left(P_{\text {ave }}=1 /\left(t_{f}-t_{i}\right) \int_{t_{i}}^{t_{f}}\left|F_{I}(t) . v(t)\right| d t\right)$ to quantify the interaction performance under different task conditions, where, $t_{i}$ and $t_{f}$ are the beginning and ending times of a phase (ramp or hold), respectively. We also inspect average dominant frequency of oscillations in interaction force $\left(\Omega=1 / N \sum_{i=1}^{N} 1 /\left|t_{\max , i}-t_{\min , i}\right|\right)$, where $t_{\max , i}\left(t_{\min , i}\right)$ represents the time at $i^{\text {th }}$ local maximum (minimum), and $N$ stands for the total number of consecutive local minima and maxima in interaction force $F_{I}$. In addition, average amplitude of oscillations in interaction force $\left(A_{F}=1 / N \sum_{i=1}^{N}\left|F_{\max , i}-F_{\min , i}\right|\right)$ and end-effector position $\left(A_{P}=1 / N \sum_{i=1}^{N}\left|x_{\max , i}-x_{\min , i}\right|\right)$ are computed, where $F_{\max , i}\left(F_{\min , i}\right)$ represents the $i^{\text {th }}$ local maximum (minimum) in interaction force $F_{I}$, and $x_{\max , i}\left(x_{\min , i}\right)$ indicates the $i^{\text {th }}$ local maximum (minimum) in end-effector position $x$.

\section{Data Analysis}

For each subject, the performance metrics were calculated for the both phases (except $A_{P}$, which is evaluated for the hold phase only) of all trials and then normalized for the
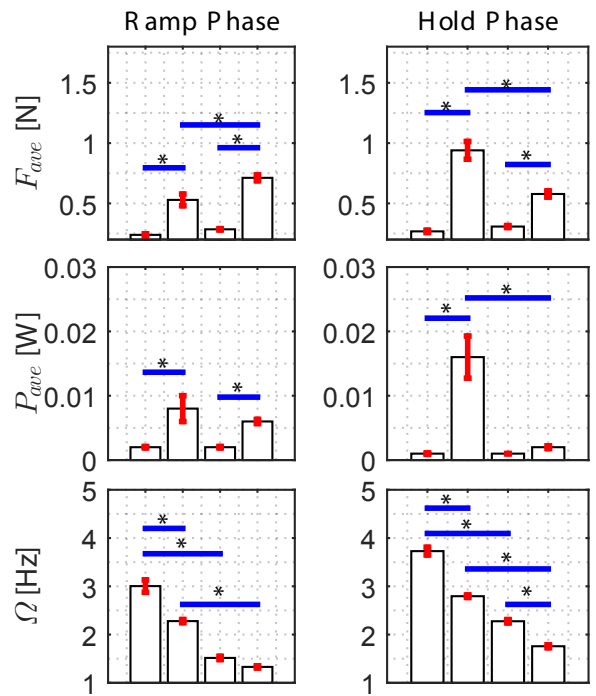

(c)
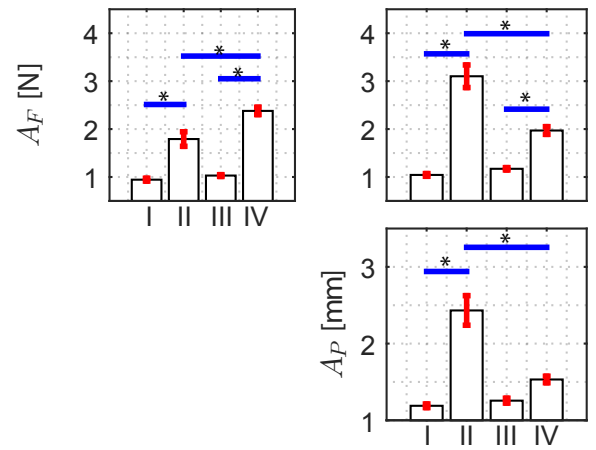

(e)

Fig. 5: The means and the standard errors of means of normalized performance metrics (I: FOAC-LAM, II: IOACLAM, III: FOAC-HAM, IV: IOAC-HAM. Horizontal bars with $*$ on top indicate statistical significance between results of the two corresponding conditions).

analysis (Fig. 5). We initially performed two-way ANOVA using SPSS software to investigate the statistical significance of these results. We observed that the admittance mass and type of controller (factors) have significant effects on the performance and there is an interaction between them. Further analysis was performed to evaluate the individual effects of admittance mass and types of controller on interaction performance. In all statistical analyses, a significance level of $\mathrm{p}=0.005$ is used to test the null hypothesis.

\section{Results}

Fig. 5a illustrates average interaction force, $F_{\text {ave }}$, during both phases. We observe a statistically significant effect of controller type on $F_{\text {ave }}$. Specifically, $F_{\text {ave }}$ is significantly lower under FOAC than that of IOAC both for HAM and LAM during both phases. $F_{\text {ave }}$ under IOAC is significantly lower with LAM (HAM) than that of HAM (LAM) during the ramp (hold) phase.

Fig. 5b demonstrates average power, $P_{\text {ave }}$, during both phases. $P_{\text {ave }}$ is significantly lower under FOAC than that of IOAC during both phases when admittance mass is low. $P_{\text {ave }}$ is significantly lower under FOAC than that of IOAC during only the ramp phase when HAM is used. The effect of change in admittance mass on $P_{\text {ave }}$ is significant under 
IOAC during only the hold phase, and $P_{a v e}$ is higher under LAM than that of HAM.

Average dominant frequency of oscillations in interaction force, $\Omega$, is illustrated in Fig. $5 \mathrm{c}$. We observe that $\Omega$ is significantly higher under FOAC than that of IOAC during both phases when LAM is used. $\Omega$ is significantly higher under FOAC than that of IOAC during the hold phase for HAM. It is also higher under LAM than that of HAM during both phases for FOAC and IOAC.

Fig. 5d shows average amplitude of oscillations in interaction force, $A_{F}$, during both phases. We observe that $A_{F}$ is significantly lower under FOAC than that of IOAC both for HAM and LAM during both phases. $A_{F}$ under IOAC is significantly lower with LAM (HAM) than that of HAM (LAM) during the ramp (hold) phase.

Average amplitude of oscillations in end-effector position, $A_{P}$, during the hold phase is shown in Fig. 5e. $A_{P}$ is significantly lower under FOAC than that of IOAC when LAM is used. $A_{P}$ under IOAC is significantly lower with HAM than that of LAM.

\section{E. Discussion}

In this experiment, we investigate the effect of FOAC on human performance in a pHRI task involving contact interactions with environment; compressing a spring with a certain rate (ramp phase) and hold there for 10 seconds (hold phase). Due to the frequency-dependent nature of damping in FOAC, the vibrations are better absorbed in both phases of the task (Fig. 5e). It is reasonable to argue that FOAC reduces these oscillations because not only it supplies more damping for higher frequencies, but also effective damping is already higher under FOAC. We experimentally examined the effect of increase in admittance mass to evaluate this argument since higher admittance mass leads to more effective damping under FOAC. However, we observe no significant change in the amplitudes of interaction force under FOAC both for LAM and HAM (Fig. 5d). Additionally, reduction in average interaction force (Fig. 5a) under FOAC indicates that human performs the task with less effort during the ramp phase.

As a downside of FOAC, since lower effective mass is rendered under FOAC, frequency of force oscillations increases slightly (Fig. 5d), and this may not be desirable during contact interactions. However, FOAC reduces the effect of increase in frequency by supplying more damping to the system, which reduces the oscillation amplitudes. For example, average amplitude of oscillations in end-effector position is significantly reduced under FOAC during hold phase (Fig. 5e). Moreover, average interaction force, average power and average amplitude of oscillations in interaction force are all reduced significantly (Fig. 5a,b,d).

\section{CONCLUSION}

We proposed FOAC for pHRI systems, and compared it with IOAC in terms of stability and performance. The stability analysis reveals that stable parameter region was enlarged, and robustness of the system for changes in environment stiffness was improved under FOAC. The experimental results showed that FOAC could bring an advantage to pHRI tasks, which involve contact interactions with environment.

Although FOAC enlarged the stability region and showed robustness to change in environment stiffness, stability analysis was conducted for a limited set of parameters and by using LTI models where time-variant behavior of human arm was neglected. A detailed and comprehensive analysis for various conditions (i.e. change in mass and damping of human model) will be conducted in the future. In addition, we will further investigate the performance and stability under FOAC when robot contacts with a spring which has a nonlinear behavior. This will show if improvement in the stability reported under FOAC also implies robustness to dynamic changes in the stiffness.

\section{REFERENCES}

[1] T. Tsumugiwa, R. Yokogawa, and K. Yoshida, "Stability analysis for impedance control of robot for human-robot cooperative task system," in IEEE/RSJ International Conference on Intelligent Robots and Systems, vol. 4, 2004, pp. 3883-3888.

[2] V. Duchaine and C. M. Gosselin, "Investigation of human-robot interaction stability using lyapunov theory," in IEEE International Conference on Robotics and Automation, 2008, pp. 2189-2194.

[3] W. Gallagher, D. Gao, and J. Ueda, "Improved stability of haptic humanrobot interfaces using measurement of human arm stiffness," Advanced Robotics, vol. 28, no. 13, pp. 869-882, 2014.

[4] J. E. Colgate and N. Hogan, "Robust control of dynamically interacting systems," International Journal of Control, vol. 48, no. 1, pp. 65-88, 1988.

[5] S. P. Buerger, H. I. Krebs, and N. Hogan, "Characterization and control of a screw-driven robot for neurorehabilitation," in IEEE International Conference on Control Applications, 2001, pp. 388-394.

[6] T. Tsuji, P. G. Morasso, K. Goto, and K. Ito, "Human hand impedance characteristics during maintained posture," Biological Cybernetics, vol. 72, no. 6, pp. 475-485, 1995. [Online]. Available: http://dx.doi.org/10.1007/BF00199890

[7] J. M. Dolan, M. B. Friedman, and M. L. Nagurka, "Dynamic and loaded impedance components in the maintenance of human arm posture," IEEE Transactions on Systems, Man, and Cybernetics, vol. 23, no. 3, pp. 698-709, May 1993.

[8] S. P. Buerger and N. Hogan, "Complementary stability and loop shaping for improved human-robot interaction," IEEE Transactions on Robotics, vol. 23, no. 2, pp. 232-244, April 2007.

[9] A. Haddadi and K. Hashtrudi-Zaad, "Bounded-impedance absolute stability of bilateral teleoperation control systems," IEEE Transactions on Haptics, vol. 3, no. 1, pp. 15-27, 2010.

[10] O. Tokatli and V. Patoglu, "Stability of haptic systems with fractional order controllers," in IEEE/RSJ International Conference on Intelligent Robots and Systems, Sept 2015, pp. 1172-1177.

[11] Y. Chen, I. Petras, and D. Xue, "Fractional order control - a tutorial," in American Control Conference, 2009, pp. 1397-1411.

[12] J. S. Mehling, J. E. Colgate, and M. A. Peshkin, "Increasing the impedance range of a haptic display by adding electrical damping," in First Joint Eurohaptics Conference and Symposium on Haptic Interfaces for Virtual Environment and Teleoperator Systems, 2005, pp. 257-262.

[13] N. Colonnese, S. M. Sketch, and A. M. Okamura, "Closed-loop stiffness and damping accuracy of impedance-type haptic displays," in IEEE Haptics Symposium, 2014, pp. 97-102.

[14] D. Matignon, "Generalized fractional differential and difference equations: stability properties and modelling issues," in Mathematical Theory of Networks and Systems symposium, 1998, pp. 503-506.

[15] F. Dimeas and N. Aspragathos, "Online stability in human-robot cooperation with admittance control," IEEE Transactions on Haptics, vol. 9, no. 2, pp. 267-278, 2016. 